\section{JTI}

JOURNAL OF

TRAUMA AND INJURY

\title{
Recurrence of Minimal Change
} Disease Following a Motor Vehicle Trauma: An Atypical Cause and Review of Literature

\author{
Jayaramakrishna Depa, M.D., George Coritsidis, M.D. \\ Department of Nephrology, Elmhurst Hospital Center, Icahn School of Medicine at Mount \\ Sinai, Elmhurst, NY, USA
}

Received: October 17, 2018

Revised: December 19, 2018

Accepted: February 7, 2019

\section{Correspondence to}

Jayaramakrishna Depa, M.D.

Department of Nephrology, Elmhurst Hospital Center, 79-01 Broadway, Queens, NY 11373, USA

Tel: $+1-718-334-3930$

Fax: +1-718-334-5160

E-mail: sonu.reddy35@gmail.com
Minimal change disease (MCD) in children has a favorable long-term prognosis, and development of end-stage renal disease is very uncommon; less than 5\%. In the first case of its kind, we report a 21-year-old female with a history of MCD at the age of 6, who had late relapse subsequent to a motorcycle accident resulting in a de-gloving skin injury and intensive care unit admission. MCD was confirmed by normal light microscopy, podocyte effacement on electron microscopy and absence of any deposits on immunofluorescence 3 weeks after the incident due to critical illness. It is postulated that the skin injury is what caused the relapse of MCD.

Keywords: Minimal change disease; Skin injury; Recurrence; Nephrology; End-stage renal disease

\section{INTRODUCTION}

Minimal change disease (MCD) in children generally has a favorable long-term prognosis, and development of end-stage renal disease is very uncommon; less than $5 \%$. In adults it accounts for $10-15 \%$ of nephrotic syndromes. Interestingly, late relapses ( $>10$ years) after a prolonged remission are not uncommon, with various etiologies associated with recurrence [1]. We report a late relapse of MCD likely sparked by the patient's trauma; a motorcycle accident that more specifically resulted in a de-gloving skin injury. To our knowledge this is the first case report of recurrence of MCD following a trauma. (http://creativecommons.org/licenses/by-nc/4.0/) which permits unrestricted noncommercial use, distribution, and reproduction in any medium, provided the original work is properly cited. 


\section{JTI}

\section{CASE REPORT}

A 21-year-old Hispanic female with history of MCD as a child at the age of 6 years was admitted to the surgical trauma intensive care unit (ICU) after falling from a motorcycle. The patient suffered extensive skin abrasions involving $40 \%$ of her body requiring extensive skin debridement. The wounds were described by surgery and emergency medicine as: "tender road rash involving specifically the thorax anteriorly and posteriorly; the intergluteal region, perineum, and shoulders bilaterally, and involving $50 \%$ of the chest, $50 \%$ of the back, $50 \%$ of the buttocks, and $25 \%$ of the legs. Full thickness wounds were present over bilateral knees and shoulders, with variable depths elsewhere. Of the extremities, the right leg, left leg, right knee, right shoulder, left arm, left shoulder, and right arm were involved with deep maceration of left knee with $-7 \mathrm{~mm}$ of exposed intact smooth bone. Computed tomography scans of the head, cervical, thoracic and lumbar spine, abdomen and pelvis, and chest were all negative and there were no fractures or head injury." The injury severity score was 1 .

Admission serum exam was the following: blood urea nitrogen $(B U N)(9 \mathrm{mg} / \mathrm{dL})$; creatinine $(0.83 \mathrm{mg} / \mathrm{dL})$, and albumin $(4.5 \mathrm{~g} / \mathrm{dL})$ all normal as was the urinalysis; negative for both protein and blood. By day 3 her albumin level was noted to have declined precipitously to $1.9 \mathrm{~g} / \mathrm{dL}$ and her urinalysis now reflected protein. On day 4, due to fever, tachycardia and an elevated white blood cell count $(15.8 \mathrm{k} / \mathrm{mcl})$, empiric vancomycin and cefepime was started and continued for 5 days. Infectious work up inclusive of urine, blood and wound cultures was negative. By day
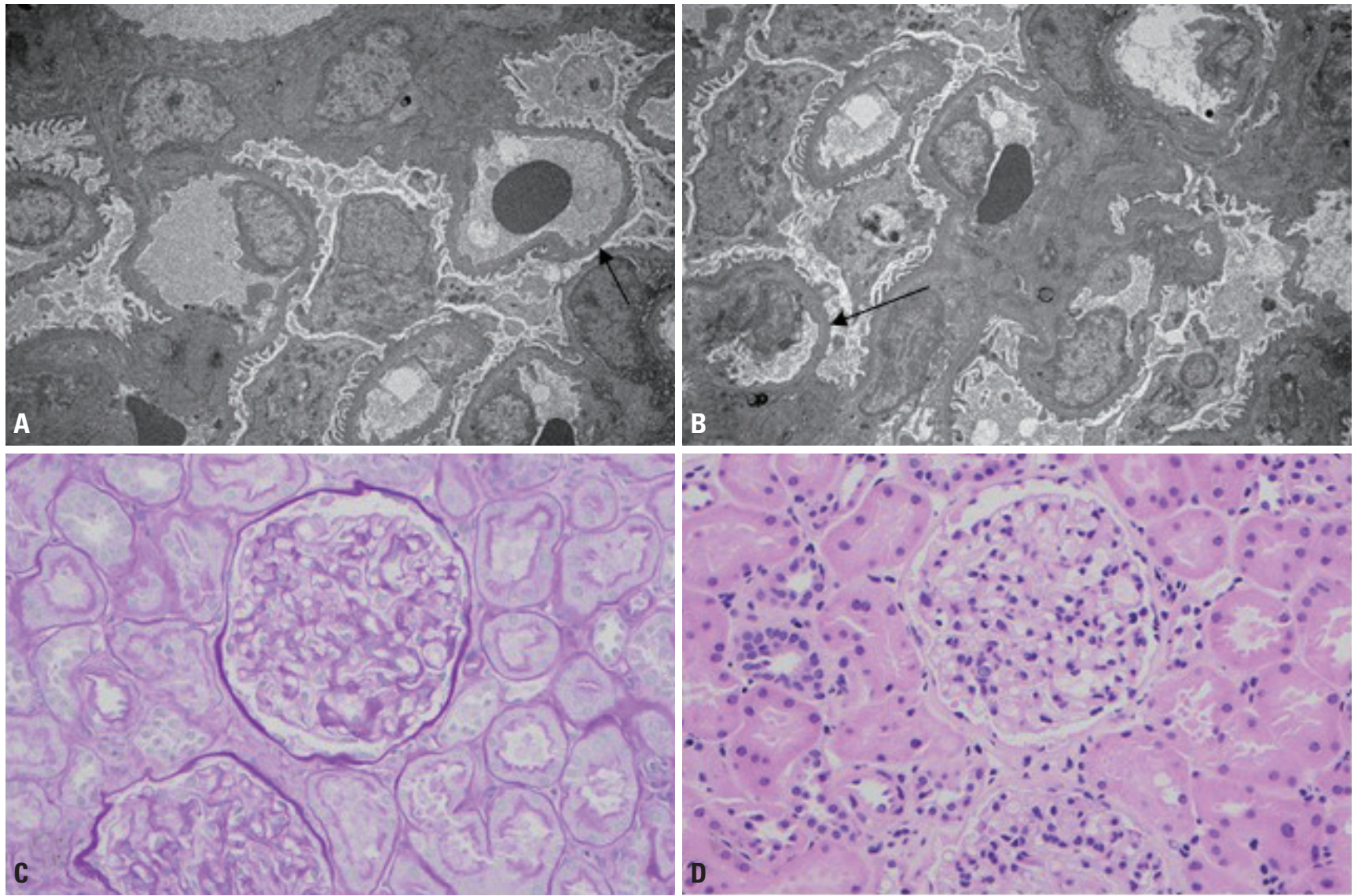

Fig. 1. (A, B) Electron microscopy results showing podocyte effacement (black arrow) (A: transitional electron microscopy, $\times 25,625$; B: transitional electron microscopy, $\times 25,625)$. (C, D) PAS and H\&E staining showing normal light microscopy (C: periodic acid-schiff, $\times 40$; D: hematoxylin and eosin, $\times 40$ ). PAS: periodic acid-schiff. 
9 diffuse leg swelling was noted along with a decreased albumin to 1.5. The urine random protein/creatinine (UPC) was found to be 10 grams and confirmed with a 24 hours urine collection indicating $10 \mathrm{~g} /$ day. Serological work up for proteinuria including anti-nuclear antibody, rheumatoid factor, and serum complements C3, C4, hepatitis profile, human immunodeficiency virus and syphilis panel were negative. An elevated serum immunoglobulin $\mathrm{E}$ (IgE) level to $960 \mathrm{IU} / \mathrm{mL}$ was noted.

On the 11th day prednisone was started at $1 \mathrm{mg} / \mathrm{kg}$ due to suspicion of MCD recurrence, and the fear of persistent wound exudation, edema, and infection as well as the heightened deep venous thrombosis risk from the hypoalbuminemic state if left prolonged. Throughout her stay the renal pathology was exclusively nephrotic syndrome without any renal insufficiency. The patient's eGFR remained $>60 \mathrm{~mL} / \mathrm{min} / 1.73 \mathrm{~m}^{2}$ until discharge.

The patient responded with decreased swelling and a repeat UPC of 0.5, five days after steroids. A biopsy was not initially performed due to critical illness but performed later as an outpatient in 3 weeks. MCD was confirmed by normal light microscopy and podocyte effacement on electron microscopy (Fig. 1). There was absence of any deposits on immunofluorescence; however, images could not be provided as the immunofluorescence slides had faded. Proteinuria had largely resolved by then.

\section{DISCUSSION}

MCD is a common cause of nephrotic syndrome; about $90 \%$ in children younger than 10 years, $50 \%$ to $70 \%$ of older children, and $10 \%$ to $15 \%$ of adults. Most cases of MCD are idiopathic (or primary) and not clearly associated with an underlying disease or event.

The exact pathogenesis of idiopathic MCD or relapse has not been fully elucidated. For some time, it has been suggested that a dysfunction of cell-mediated immunity, namely, an abnormal clone of T-cells, may result in the production of cytokines or circulating factors altering the glomerular filtration barrier leading to proteinuria [2]. More recently the focus has included CD80 podocyte abnormalities [3].

Late relapses in adulthood are widely variable from
$5-42 \%$ [4]. Majority of studies concluded that among the risk factors of relapses during adulthood are a young age at onset $<6$ years, a high number of relapses during childhood, and the use of alkylating agents and cyclosporine.

Relapses and or recurrences have also been linked to numerous triggers: neoplastic, drugs, infections, allergies, as well as atopic disorders where elevated serum IgE levels have been noted $[5,6]$. Multiple allergens have been reported in association with MCD, including fungi, poison ivy, ragweed and timothy grass pollen, house dust, medusa stings, bee stings, vaccinations and cat fur. In fact, $30 \%$ to $40 \%$ of children with steroid-sensitive nephrotic syndrome have some type of allergic disorder or asthma [7].

We believe that the resulting skin injury rather than the trauma per se initiated the MCD, possibly IgE mediated. In other skin injuries, such as from burns, glomerular pathology has been implicated but as IgA nephropathy not MCD [7]. Our patient was found to have an elevated IgE level of $960 \mathrm{IU} / \mathrm{mL}$. Many patients with MCD have increased serum IgE and interleukin (IL)-13 levels. IL-13 has been shown to induce B cell IgE production, podocyte CD80 expression and proteinuria [8,9]. Urinary CD80 levels are increased in patients with MCD during relapse and return to normal after remission.

In our patient proteinuria was recognized within 3 days of the injury. There were no urinary abnormalities on admission, or evidence of infection during the admission. The fever and tachycardia may have been symptoms of the inflammatory condition of trauma, and all cultures proved to be negative. Antibiotics were initiated within 4 days of the injury making them less likely the etiology. Initial medications (listed) are not considered allergenic (IV crystalloids, hydromorphone as needed for pain and local dressings for wounds). The patient denied a history of allergies or the use of recent antibiotics. In short neither infection, nor allergic reaction to medications is a likely explanation for the recurrence.

Trauma directly influences GFR by augmentation, assuming normal renal function, and the patient's proteinuria may have been enhanced by such changes in glomerular hemodynamics [10]. Trauma is also a systemic inflammatory event with immunological consequences also likely influencing this presentation [11]. Even so MCD has not been previously described in trauma. We 
therefore postulate that skin inflammation caused by extensive road rash along with the inflammatory response of trauma could have triggered the inflammatory cascade that led to the relapse of MCD.

\section{REFERENCES}

1. Skrzypczyk P, Panczyk-Tomaszewska M, Roszkowska-Blaim M, Wawer Z, Bienias B, Zajgzkowska M, et al. Long-term outcomes in idiopathic nephrotic syndrome: from childhood to adulthood. Clin Nephrol 2014;81:166-73.

2. Gkrouzman E, Kirou KA, Seshan SV, Chevalier JM. Minimal change disease as a secondary and reversible event of a renal transplant case with systemic lupus erythematosus. Case Rep Nephrol 2015;2015:987212.

3. Ishimoto T, Cara-Fuentes G, Wang H, Shimada M, Wasserfall $\mathrm{CH}$, Winter WE, et al. Serum from minimal change patients in relapse increases CD80 expression in cultured podocytes. Pediatr Nephrol 2013;28:1803-12.

4. Hjorten R, Anwar Z, Reidy KJ. Long-term outcomes of childhood onset nephrotic syndrome. Front Pediatr 2016;4:53.
5. Tan Y, Yang D, Fan J, Chen Y. Elevated levels of immunoglobulin E may indicate steroid resistance or relapse in adult primary nephrotic syndrome, especially in minimal change nephrotic syndrome. J Int Med Res 2011;39:2307-13.

6. Shao YN, Chen YC, Jenq CC, Hsu HH, Chang MY, Tian YC, et al. Serum immunoglobulin $\mathrm{E}$ can predict minimal change disease before renal biopsy. Am J Med Sci 2009;338:264-7.

7. Zhu J, Wang H, Yang D. IgA Nephropathy with pathologic features of membranoproliferative glomerulonephritis following burn injury. Case Rep Nephrol Urol 2014;4:31-6.

8. Abdel-Hafez M, Shimada M, Lee PY, Johnson RJ, Garin EH. Idiopathic nephrotic syndrome and atopy: is there a common link? Am J Kidney Dis 2009;54:945-53.

9. Ceri M, Kurultak I. Relapse of nephrotic syndrome after a bee sting. Indian J Nephrol 2012;22:151-2.

10. Udy AA, Roberts JA, Shorr AF, Boots RJ, Lipman J. Augmented renal clearance in septic and traumatized patients with normal plasma creatinine concentrations: identifying at-risk patients. Crit Care 2013;17:R35.

11. Stoecklein VM, Osuka A, Lederer JA. Trauma equals danger-damage control by the immune system. J Leukoc Biol 2012;92:53951. 